\title{
LE TRASFORMAZIONI BIRAZIONALI DELLO SPAZIO INERENTI AD UNA CUBICA SGHEMBA.
}

Nota di Grazia Macrina Caldarera, in Catania.

Adunanza del I4 febbrajo 5904 .

Sono oggetto del presente studio le più generali trasformazioni Cremoniane dello spazio, involutorie e no, che hanno le coppie di punti omologhi distribuite univocamente sulle $\infty^{3}$ rette incidenti una cubica sghemba data. Si tratta cioè della generazione e dei caratteri projettivi di alcune corrispondenze birazionali fra i punti dello spazio, le quali hanno a sostegno un complesso cubico speciale di rette, e si presentano immedratamente dopo quelle determinate da complessi lineari e quadratici ${ }^{*}$ ).

\section{La trasformazione $T$.}

I. Sia data una cubica gobba $p^{3}$, ed indichiamo con $\Gamma$ il complesso di tutte le rette incidenti alla medesima. Consideriamo due spazi sovrapposti $\Sigma$ e $\Sigma^{\prime}$ riferiti algebricamente fra loro secondo una corrispondenza biunivoca non involutoria, che chiameremo $T$, la quale abbia le sue $\infty^{3}$ coppie di punti distribuite sugli $\infty^{3}$ raggi di $\Gamma$, per modo che ciascun raggio di $\Gamma$ contenga una e (generalmente) una sola coppia di $T$. Siano $R, R^{\prime}$ le due rappresentazioni prospettive di $\Gamma$ su $\Sigma$ e $\Sigma^{\prime}$, che nascono

*) Vedı Montesano, Rendiconti Accademia Napoli, volume $\mathrm{II}_{2}$; Rendiconti Accademia Lincei, volume $\mathrm{IV}_{4}$ e $\mathrm{V}_{4}$; Rendiconti Istituto Lombardo I892.-PIERI, Rendiconti del Circolo Matematico di Palermo, volume VI e VII. 
da $T$ : onde $T=R^{-\mathrm{r}} R^{\prime}, T^{-\mathrm{r}}=R^{\prime-\mathrm{r}} R$. Supporremo che $T$ sia la piu generale fra le trasformazioni birazionali non involutorie determinate, nel modo anzidetto, dal complesso cubico $\Gamma$.

Il complesso $\Gamma$ contiene $\infty^{2}$ congruenze $\gamma$ del primo ordine e terza classe, ognuna delle quali consta di rette appoggiate simultaneamente alla cubica $p^{3}$ e ad una $c$ delle sue corde. Di sifatte congruenze due qualunque hanno a comune una schiera rigata di $\Gamma$, costituita dalle rette che si appogziano simultaneamente alla cubica e a due delle sue corde; e tre qualunque non hanuo, in generale, nessun raggio in comune.

Le coppie do punti omologhi in $T$, che stan no sui raggi do una congruenza $\gamma \equiv p^{3} c$ ( $c$ designando una corda della $p^{3}$ ) generano due superficie omologhe $\chi$ e $\chi^{\prime}$ del $4^{\circ}$ ordine, le quali passano semplicemente per la corda $c$ e contengono come linea doppia la cubica $p^{3}$. Queste due superficie saranno pertanto rigate razional del $4^{\circ}$ grado; e al variare di $c$ descriveranno rispettivamente due reti $[\chi]$ e $\left[\chi^{\prime}\right]$.

Le due superficie $\chi$ e $\chi^{\prime}$ inerenti ad una stessa congruenza $\gamma \equiv p^{3} c$, sono tagliate dalla quadrica $\rho^{2}$, sostegno di una schiera rigata di questa, secondo due rette $r$ ed $r^{\prime}$ sghembe e direttrici della scbiera.

Se la corda $c$ descrive una schiera rigata $\rho^{2}$, la superficie $\chi$, ad essa inerente, descrive un fasciv $(\chi)$. Variando $\rho^{2}$, questi fasci (che sono $\infty^{2}$ in tutto come le $\rho^{2}$ ) ci daranno tutti i fasci contenuti nella rete $[\chi]$. Inoltre due qualunque fra le $\infty^{2}$ superficie $\chi$ provengono sempre da due corde $c$ di $p^{3}$, le quali sono generatrici di una determinata schiera $p^{2}$.

Tutte le superficie del fascio $(\chi)$ inerente alla schiera $\rho^{2}$ passeranno per la retta $r$ direttrice comune alla $\rho^{2} e$ ad una qualunque di esse. La base del fascio $(\chi)$ è composta della $p^{3}$ contata quattro volte, della $r$ contata una volta, e di un luogo residuo $o^{3}$, formato da tre corde $o_{I}$, $o_{2}, o_{3}$ della cubica $p^{3}$, nessina delle qual puo giacere sulla quadrica $p^{2}$.

Un luogo analogo $o^{\prime}{ }^{\prime}$ si offre nello spazio $\mathbf{s}^{\prime}$.

Tutte le superficie delle reti $[\chi]$ e $\left[\chi^{\prime}\right]$ passano rispertivamente per $0^{3}$ ed $0^{\prime 3}$, e si potrebbe vedere in pù̀ modi che la rete delle $\chi$ risulta pienamente determinata dalla cubica $p^{3}$, che è direttrice doppia per tutte, e dalle tre corde $o_{1}, O_{2}, O_{3}$ di questa, che sono generatrici comuni.

2. I due fasci $(\chi)$ e $\left(\chi^{\prime}\right)$ sono riferiti univocamente fra loro, essendo omologhe due superficie $\chi$ e $\chi^{\prime}$ inerentı ad una medesima generatrice $c$ di $\rho^{2}$; e l'intersezione variabile di due superficie omologhe, spo- 
gliata di una generatrice $c$ di $\rho^{2}$, sarà un luogo di punti uniti per $T$. Onde: La trasformazione $T$ ammette ura superficie punteggiata unita $\sigma$ del $6^{\circ}$ ordine, che passa semplicemente per entrambe le linee $0^{3}$ ed $0^{\prime}{ }^{3} e$ contiene la $p^{3}$ come curva tripla. La superficie $\sigma^{6}$ è una rigata ellittica.

3. Se $L$ è un punto di $p^{3}$, la sezione del piano $L c$ ( $c$ essendo una corda qualunque di $p^{3}$ ) sulle superficie $\chi^{4}$ e $\chi^{\prime 4}$ si comporrà, tolta via questa retta, di due curve descritte dai punti omologhi di $\Sigma$ e $\Sigma^{\prime}$ che stanno sui raggi del fascio $L c$. Pertanto : le cuppue da punti corrispondenti in $T$, che stanno sui raggi di ina stella $(L)$, di $\Gamma$, generanu due superfacre cubrche $\lambda$ e $\lambda^{\prime}$, avente un punto doppio in $L$ e passanti semplicemente per la $p^{j}$.

Visto che ad un punto $A$ di $o^{3}$ corrispondono, in forza di $R^{-1}$, tutti i raggi che da esso prolettano $p^{3}$, bisognerà che tulte le superficte $\lambda$ passino semplicemente per il luogo $0^{3}$. Analogamente le $\lambda^{\prime}$ contengono come semplici le tre rette $o_{1}^{\prime}, o_{2}^{\prime}, o_{3}^{\prime}$. Al variare di $L$ su $p^{3}$ le superficie $\lambda^{3}$ e $\lambda^{\prime 3}$ descrivono due fasci $(\lambda)$ e $\left(\lambda^{\prime}\right)$. La base di ciascuno di tali fasci si comporrà della cubica $\hat{p}^{3}$ contata due volte (giacchè tutte le $\lambda$, e cosi pure le $\lambda^{\prime}$, dovranno toccarsı lungo $p^{3}$ ) insieme con le tre corde $o_{\mathrm{r}}$, $o_{2}, o_{3}$, ovvero $o_{1}^{\prime}, o_{2}^{\prime}, o_{3}^{\prime}$ rispettivamente.

Mediante i due fasci $(\lambda)$ e $\left(\lambda^{\prime}\right)$, univocamente riferiti alla punteggrata cubica $(L)$, e quindi proiettivi fra loro, risulta individuata la trasformazione $T$; la superficie punteggiata unita $\sigma^{6}$ viene ad essere quella generatal da codesti due fasci.

La trasformazione $T$ può anche venire costruita come segue. $\mathrm{S}_{1}$ osservi che le due retı $[\chi]$ e $\left[\chi^{\prime}\right]$ risultano riferite proiettivamente fra loro in virtù di $T$. Ora se la rete di superficie del $4^{\circ}$ ordine contenenti la cubica $p^{3}$ come doppia e le rette $o_{1}, o_{2}, o_{3}$ come semplici si riferisce prolettivamente alla rete analoga determinata da $p^{3}$ con le rette $o_{1}^{\prime}, o_{2}^{\prime}$, $o_{3}^{\prime}$, avremo che al fascio di superficie dell'una rete contenenti un punto $A \mathrm{~d}_{1} \Sigma$ (e quindi anche la corda $r$ di $p^{3}$ che parte da quel punto) corrisponderà un fascio analogo dell'altra rete, della cui base farà parte un'altra corda $r^{\prime}$ di $p^{3}$. Ora, conducendo per $A$ quell'unica retta che incontra al medesimo tempo la cubica $p^{3}$ e la retta $r^{\prime}$ (in punti distinti); e chramando $A^{\prime}$ il suo punto d'incontro con $r^{\prime}$, verremo a definire fra lo spazio $\Sigma$ descritto da $A$ e lo spazio $\Sigma^{\prime}$ descritto da $A^{\prime}$ una corrispondenza birazionale della specie $T$ : anzi (ove la proiettıvita stabilita fra le 
due reti sia del tutto generica) la più generale possibile di questa specie. L'osservazione prova altresi che in virtu di $T$ ogni corda di $p^{3}$ si trasforma punto per punto, cioe proiettivamente, in un'altra corda di $p^{3}$.

Le $o^{3}$ ed $o^{\prime 3}$ sono linee fondamentali per $T$ e $T^{-1}$ rispettivamente, nonchè per le $R^{-1}$ ed $R^{\prime-1}$.

Si osservi che, fissato un monoide $\lambda$ (o $\left.\lambda^{\prime}\right)$, le tre rette che, partendo dal suo centro, $L$, si appoggiano a due delle tre rette 0 (od $o^{\prime}$ ) giacciono evidentemente sul monoide stesso; si hanno cosi tre delle sei rette del monoide uscenti dal suo punto doppio; le altre non incontrano le rette $o\left(\operatorname{od} o^{\prime}\right)$.

4. La superficie $\varphi^{\prime}$, che in forza di $T$ corrisponde ad un piano generico $\varphi$ di $\Sigma$, taglierd questo piano nelle tre cubiche annesse ai fasci di rette di $\Gamma$ giacenti sul medesimo e nella traccia di esso piano sulla superficie unita $\sigma^{6}$. Pertanto: La $T$ e la sua inversa sono entrambe del I $5^{\circ}$ ordine e per esse la $p^{3}$ sarà fondamentale settupla ed il luogo ó (ovvero rispettivamente o) fondamentale quadruplo. D'altra parte, come vedremo in breve, a ciascun punto della $p^{3}$, considerata in $\Sigma^{\prime}$, corrisponde in $\mathbf{\Sigma}$ una curva mobile del $6^{\circ}$ ordine: per la qual cosa le $\infty^{3}$ superficie $\varphi^{\prime}$ dovranno avere una delle selte falde in comune lungo tutta la cubica $p^{3}$. Inoltre è facile vedere che questa falda è anche comune a tutte quante le superficie $\lambda^{\prime}$.

Essendo $o_{1}, O_{2}, o_{3}$ le rette, di cui si compone $o^{3}$, se una retta $u-$ scente da un punto $L$ di $p^{3}$ si appoggia ad $o_{1}, o_{2}$, per esempio, giacierà sulla superficie $\lambda$ inerente a quel punto, e corrisponderà per intero alla sua traccia in $\lambda^{\prime}$ : traccia che sarà dunque un punto unito per $T$, vale a dire un punto di $\sigma^{6}(\$ 2)$. Il luogo di questo punto è una retta $d_{r, 2}^{\prime}$, corda di $p^{3}$. Altre due rette analoghe $d_{2,3}^{\prime}, d_{3,1}^{\prime}$, si offriranno del pari in $\Sigma^{\prime}$.

Le tre rette $d_{1,2}^{\prime}, d_{2,3}^{\prime}, d_{3, \mathrm{r}}^{\prime}$ sono fondamentali semplici in $T^{-1}$; ad esse corrispondono rispettivamente in $\Sigma$ le rigate quadriche $\delta_{1,2}, \delta_{2,3} \delta_{3,1}$, determinate dalla curva $p^{3}$ e dalle rette $o_{1}, o_{2}, o_{3}$, prese a due a due. Analogamente si hanno in $\Sigma$ tre rette $d_{1,2}, d_{2,3}, d_{3,1}$ fondamentali semplici in $T$. Nuove linee fondamentali potranno aversi come segue: Indichiamo con $b_{\mathrm{r}}, b_{2}, b_{3}$ le tre corde di $p^{3}$ uscenti dal punto $L$ e giacenti sulla superficie $\lambda^{3}$ che ha questo punto per centro. Poichè ciascuna di queste rette si appoggia ulteriormente a $p^{3}$ non potrd incontrare la 
superficie $\lambda^{\prime}$, corrispondente di $\lambda$, che nel punto $L$ e nell'ulteriore suo punto di appoggio a $p^{3}$. Esisterà dunque un numero finito di punti $L$, pei ognuno dei quals una delle anzidette corde $b$ appartiene tutta quanta alla superficie $\lambda^{\prime}$. Una tal corda sarà parassita per $T$ e $T^{-1}$; e quind fondamentale seinplice per ambedue le trasformazioni.

Il numero di queste rette parassite può aversi come segue. Sia, per esempio, $n$ il piano tangente in un punto $L$, di $p^{3}$ a tutte le superficie $\lambda$ ed $\eta^{\prime}$ il piano tangente a tutte le $\lambda^{\prime}$. Se questi due piani coincidono in $n$, allora le due superficie $\lambda_{L}$ e $\lambda_{L}^{\prime}$, inerenti al punto $L$ dove il piano $n$ taglierà la cubica fuori di $L_{1}$, contengono tutta la retta $L L_{1}$. Viceversa, se due superficie $\lambda_{M}, \lambda_{M}^{\prime}$ devono aver $M M_{1}$ di $p^{3}$, bisognerà evidentemente che esse abbiano lo stesso piano tangente in $M_{1}$. Onde le rette cercate saranno tante, quanti sono i punti di $p^{3}$ in ciascuno dei quali le superficie $\lambda$ toccano le $\lambda^{\prime}$. Ora si facciano corrispondere fra loro due punti $A$ e $A^{\prime}$ della cubica, ogni qual volta essi gracciano nei due pianı tangenti rispettivamente a tutte le $\lambda$ e $\lambda^{\prime}$ in uno stesso punto di $p^{3}$. Sulla $p^{3}$ resta cosi stabilita una corrispondenza $(3,3)$ di Chasles, con sei punti uniti; per ognuno dei quali avremo una retta parassita della trasformazione. Talı rette parassite le indicheremo in seguito con $b_{1}, b_{2} \ldots, h_{6}$.

Si vedrà in seguito $(\$ 8)$ che tutte le superficie $\varphi^{\prime}$ si osculano con una falda lungo la cubica $p^{3}$; succhè la base del sistema lineare delle $\varphi^{\prime}$ sarà pienamente assegnata con le linee $p^{3}, o^{\prime}{ }^{3}, d_{h, k}^{\prime}$ ed $h_{2}(h, k=1,2,3$, $i=\mathrm{r}, \ldots, 6)$.

Invero abbiamo:

$$
\text { I5. IS }-7 \cdot 7 \cdot 3-4 \cdot 4 \cdot 3-3-3-6=15 \text {. }
$$

5. Il cono che oscula nel punto doppio $L$ la superficis $\lambda$ inerente al medesimo, sega $\lambda^{\prime}$ lungo una sestica razionale $l^{\prime 6}$, che ha come quadruplo il punto $L$, si appoggia un due punt alle $o_{2}^{\prime}(\imath=1,2,3)$ ed in tre punti, fuori da $L$, alla cubica $p^{3}$. La detta sestica $l^{\prime 6}$ è la linea corrispondente, in forza di $T$, allintorno del punto fondamentale $L$.

Al variare di $L$ su $p^{3}$, il detto cono osculatore descrive un sistema semplicemente infinito razionale d'indice quattro. Pertanto alla cubica fondamentale $\hat{p}^{3}$ di $\Sigma$ corrisponde in $\Sigma^{\prime}$ una superficue, che chiameromo $\pi^{\prime}$, $d^{\prime}$ ordine quattordicu, luogo di tutte le sestuche $l^{\prime 6}$. [La $\pi^{\prime}$ viene infatti generata da due sistemi razionali $\infty^{x}$ di superficie, uno dei quali $(\lambda)$ d'or- 
dine 3 ed indice 1 , l'altro, quello dei coni osculatori, d'ordine 2 ed indice 4 ].

Cerchiamo ora la molteplicita di $p^{3}$ per la superficie $\pi^{14}$. Basta perciò considerare, ad esempio, l'intersezione tra $\pi^{\prime}$ e $\delta_{I 2}^{\prime}$, che deve spezzarsi nella $p^{3}$, contata $x$ volte, se $x$ è la molteplicità domandata, nelle rette $o_{1}^{\prime}, o_{2}^{\prime}$ contata quattro volte e nelle due generatrici $d_{1} \delta_{r, 2}^{\prime}$ corrispondenti ai due puntr, dove la retta $d_{r_{2}}$ si appoggia a $p^{3}$. Si trova cosi che $x=6$; cioè che la $p^{3}$ è sestupla per la superficie $\pi^{\prime}$.

Alla superficie $\pi^{\prime}$ spettano ancora come semplici le tre rette $d_{\mathrm{r}, 2}^{\prime}$, $d_{2,3}^{\prime}, d_{j, 1}^{\prime}$ e come quadruplo il luogo $o^{\prime 3}$.

6. Se $M$ è un punto di $o_{\imath}(i=1,2,3)$, sopra ogni generatrice del cono $M p^{3}$ giace un punto $M^{\prime}$ corrispondente ad $M$; ed il luogo di esso punto è una certa curva $m_{1}^{\prime}$ del $4^{\circ}$ ordine. Al variare di $M$ su $o_{1}$, la $m_{2}^{\prime}$ descrive una superficie $\omega_{2}^{\prime}$ corrispondente ad $o_{2}$. Questa superficie contiene come doppia la cubica $p^{3}$ e come semplici le tre rette $o_{l}^{\prime}(l=1,2,3)$, le due $d_{1, l}^{\prime}$ e la $o_{1}$. Ne vien facilmente che la $\omega_{2}^{\prime}$ sarà del $4^{\circ}$ ordine. Infine la $\omega_{2}^{\prime}$ non è altro che una superficie $\chi^{\prime}$ della rete $\left[\chi^{\prime}\right]$; e precisamente la superficie $\chi^{\prime}$ dovuta alla congruenza $\gamma \equiv p^{3} o_{\imath}$. Superficie come $\omega_{\imath}^{\prime}$ ne abbiamo tre in $\Sigma^{\prime}$ ed altre tre analoghe in $\Sigma$.

7. Il fatto che le curve $l^{6}(\$ S)$ corrispondenti ai punti di $p^{3}$ sono del $6^{\circ}$ ordine, mentre la curva $p^{3}$ è fondamentale settupla $(\$ 4)$, ci avverte che la superficie $\pi^{\prime 14}$, luogo delle sestiche $l^{\prime 6}$, sarà soltanto una parte del luogo corrispondente a $p^{3}$. Ciò che resta del luogo è una nuvva superficie $\xi$, la quale corrisponde in $\Sigma^{\prime}$ all'intorno di $p^{3}$ sulle superficue $\lambda$. A ciascun punto $d_{1}$ quest'intorno (o striscia infinitesima) corrisponde una retta; e cioè la retta congiungente il punto $L_{\mathrm{I}}$ di $p^{3}$, infinitamente vicino al punto che si considera, col punto $L$, dove 11 piano tangente alle $\lambda^{\prime}$ nel punto $L_{\mathrm{r}}$ sega ulteriormente la cubica. La superficie $\xi^{\prime}$ può pertanto venir generata dall'intersezione dei monoidi $\lambda^{\prime}$ con i rıspettivi coni tangenti nei centri dei monoidi stessi, quando per altro si escludano le tre quadriche $\delta_{1,2}^{\prime}, \delta_{2,3}^{\prime}, \delta_{3,1}^{\prime}$. Invero il cono che oscula nel punto doppio $L$ la superficie $\lambda_{L}^{\prime}$, sega questa lungo sei rette, tre delle quali si appoggiano ognuna a due delle $o_{1}^{\prime}, o_{2}^{\prime}, o_{3}^{\prime}$, descrivendo le tre quadriche $\delta_{\mathrm{r}, 2}^{\prime}$, $\delta_{2,3}^{\prime}, \delta_{3,1}^{\prime}$, mentre le altre tre generano la superficie rigata $\xi^{\prime}$. Ricordando che il sistema di questi coni osculatori è d'indice quattro, si ha dunque 
che: all'intorno della cubuca $p^{3}$, su tutte le superficte $\lambda$, corrisponde in $z^{\prime}$ una superficie $\xi$ ' dell'ottavo ordine. Analogamente avremo in $\Sigma$ una superficie $n^{8}$, corrispondente all'intorno $d i p^{3}$ su tutte le superficie $\lambda^{\prime}$.

Per un punto $L$ qualsiasi della curva $p^{3}$ passano quattro generatrici della rigata $n^{8}$ : tre sono quelle che giaccione sul monoide $\lambda_{L}$, la quarta appartiene al monoide $\lambda_{M}$, essendo $M$ l'ulteriore intersezione di $p^{3}$ con il piano tangente in $L$ a tutte le $\lambda$.

8. Se una retta $r$ di $\Sigma$ incontra, p. es., la generatrice $L L_{\mathbf{x}}$ della rigata fondamentale $n^{8}$ [essendo $L L_{\mathrm{I}}(\$ 7)$ la retta corrispondente al punto infinitamente vicino ad $L_{\mathrm{r}}$ sulla retta $L_{\mathrm{r}} M$, dove $M$ è l'ulteriore intersezione $\mathrm{d}_{1} \dot{p}^{3}$ col piano tangente in $L_{\mathrm{r}}$ a tutte le $\left.\lambda^{\prime}\right]$, la curva corrispondente $r^{\prime \text { is }}$ passerà per 1 punto $L_{\mathrm{r}}$ toccando quivi la retta $L_{\mathrm{r}} M$; questo passaggio implica dunque per la $r^{\prime}$ tre condizioni. Ne viene che le otto incidenze di una $r^{15}$ con la $p^{3}$, dovute alla superficie $n^{8}$, contano per 24 condizioni lineari cui deve soddisfare la $r^{\prime \prime s}$. Con ciò resta provato che il peso totale delle condizioni fondamentali, cui va soggetta ogni curva $r^{\prime 15}$, è 56 ; come doveva appunto accadere:

$$
4(\mathrm{I} 5-\mathrm{I})=\mathrm{I} 4+3.8+3 \cdot 4+3.2 .
$$

Osservando ora che la curva d'intersezione variabile fra due superficie $\varphi^{\prime \prime 5}$ è obbligata a toccare otto volte le superficie $\lambda^{\prime}$ lungo la cubica $p^{3}$, e sempre sotto una direzione determinata ed unica per ciascun punto $d \imath p^{3}$, si deduce che le $\infty^{3}$ superficie $\varphi^{\prime \prime s}$ devono oscularsi fra loro lungo $p^{3}$ con quella falda che è tangente alle superficie $\lambda^{\prime}$ ( $\mathrm{d}_{1}$ modo che la valenza di $p^{3}$ nell'intersezione di due superficie ' $\rho^{\prime}$ è $7 \cdot 7+2$ ).

Come riprova si osservi che una curva $r^{15}$, dovuta all'intersezione di due $\varphi^{\prime}$ qualunque, è effettivamente incontrata da una terza superficie $\varphi^{\prime}$, fuori dalle linee fondamental, in un solo punto variabile:

$$
\text { I S. IS }-[14 \cdot 7+8(7+2)+3 \cdot 4 \cdot 4+3 \cdot 2]=\text { I. }
$$

L'intersezione dei due coni osculatori in $L$ alle superficie $\lambda_{L}$ e $\lambda_{L}^{\prime}$ consta della tangente in $L$ a $p^{3}$ e di tre rette, le qual saranno tangenti in quel punto tanto alla sestica $l^{\prime 6}$, corrispondente ad $L$, quanto alla curva punteggiata unita che è comune alle due superficie $\lambda_{L}$ e $\lambda_{L}^{\prime}$. Dunque: la superficie $\pi^{\prime \mathbf{I} 4}$ tocca, con tre delle sue falde passanti per $p^{3}$, le tre falde della superficie unita $\sigma^{6}$ lungo tutta questa curva. Inoltre, poichè tutti gli altri rami, che la curva $l^{\prime 6}$ possiede nell'intorno di $p^{3}$, toccano le super- 
ficie $\lambda^{\prime}$, avremo ancora che tutte e tre le altre falde di $\pi^{\prime}$ passanti per $p^{3}$, toccano lungo $p^{3}$ le superficie $\lambda^{\prime}$.

Si osserverd che le sei rette parassite $b_{1}, b_{2}, \ldots, b_{6}$ semplici per la superficie fondamentale $\pi^{\prime 14}$, e semplici del pari per la superficie $\xi^{\prime 8}$, risultano doppie pertanto rispetto al luogo che corrisponde in $\Sigma^{\prime}$ alla cubica $p^{3}$, come deve essere infatti, trattandosi di rette parassite che tagliano due volte la curva $p^{3}$. Tutto cio viene confermato dall'esame dell'intersezione totale fra le superficie $\pi^{\prime 4}$ e $\sigma^{6}$.

\section{La trasformazione $I$.}

9. Passiamo ora allo studio della trasformazione involutoria $I$, che ha per sostegno il complesso cubico $\Gamma(\$ \mathrm{I})$, restringendoci sempre al caso più generale.

Sia $c$ una corda di $p^{3}$ e consideriamo la congruenza $\gamma \equiv p^{3} c$. Le $\infty^{2}$ coppie di punti coniugati, che stanno sui raggi della congruenza $\gamma$, formano una superficte autoconumgata $\chi$ del $6^{\circ}$ grado con la $p^{3}$ per linea tripla; dunque una rigata elluttuca che ammette $c$ come generatrice semplice.

I punti coniugati giacenti sui raggi di un fascio $L c$ di $\gamma$ generano una quintuca dotata di un punto triplo in $L$ e di due punti doppi negli estremi della corda $c$. Ne segue che: le coppie di punti coniugati nella $I$, che stanno sui raggi di una stella $L$ del complesso $\Gamma$, formano una superficie autoconiugata $\lambda$ del quinto ordine, avente il punto $L$ della $p^{3}$ come triplo e questa cubrca come linea doppia.

Queste superficie $\lambda$ sono omalordi; i punti di una qualunque di esse sono riferiti biunivocamente al sistema deile corde di $p^{3}$. Al variare d1 $L$ su $p^{3}$, la superficie $\lambda$ genera uni fascio; cosicchè l'involuzione $I$ è generabile mediante un fascio di superficie autocontugate $\lambda^{5}$, ognuna delle quali ba un punto triplo variabule lungo la cubica $p^{3}$ e passa (in conseguenza) due volte per questa linea, toccando le due falde di ogni altra superficie del fascio lungo tutta la medesima.

Due superficie $\lambda$ qualunque avranno a comune, oltre della $p^{3}$ contata sei volte, una linea del settimo ordine, che chiameremo $o^{7}$, formata $\mathrm{da}$ 7 corde della cubica $p^{3}$ ). Ad un punto qualunque $M$ di $o^{7}$ corrispon-

*) Le superficie del $5^{\circ}$ ordine, dotate di una cubica doppia e di un punto triplo (su di essa), furono partıcolarmente studiate da A. DEL RE in diverse Note pubblicate 
dono, per la rappresentazione di $\Gamma$ su $\Sigma$, tutte le generatrici del cono $M p^{3}$.

Essendo $c$ una corda di $p^{3}$, e $\chi$ la superficie ad essa inerente, la retta $c$ segherd ulteriormente il cono cubico $M p^{3}$ in un sol punto $N$. Sulla retta $M N$ giace intanto il coniugato $M^{\prime}$ di $M$; quind 1 la $\chi^{6}$ dovrà contenere $M$; e per conseguenza tutte le superficie $\chi^{6}$ passeranno per $0^{7}$.

Due qualunque superficie $\chi$, siano per es. $\chi_{1}$ e $\chi_{2}$, si segano nella $p^{3}$ contata nove volte, nella $0^{7}$ ed in un luogo residuo del $2^{0}$ ordine, evidentemente composto di due corde della cubica.

Le superficie $\chi$ formano complessivamente una rete, la quale è completamente determinata dalla cubica $p^{3}$, come curva base tripla, e dalle sette corde di $p^{3}$ che costituiscono $a^{7}$, come linee basi semplici.

Le $\chi$ che contengono una data corda $r$ di $p^{3}$ formano un fascio, la base del quale consta di $p^{3}$, contata nove volte, del luogo $0^{7}$ e di un'altra corda di $p^{3}$ generatrice comune a tutte le superficie del fascio e, per conseguenza, coniugata ad $r$ in forza $\mathrm{d} i \mathrm{I}$. Dunque la $I$ coordina a qualsivoglia corda $r$ di $p^{3}$ un'altra corda $r^{\prime}$; di guisa che i punti omologhi sulle due corde coniugate $r$ ed $r^{\prime}$ si corrispondono proiettivamente. Da ciò si deduce una nuova costruzione di $I$, visto che il fascio delle superficie $\chi$, contenenti un punto $A$ dato a piacere, passano tutte per la corda $r$ di $p^{3}$ che passa per $A$; e che questo fascio determina la corda $r^{\prime}$, coniugata di $r$, su cui deve stare il punto $A^{\prime}$ coniugato di $A$. Il punto $A^{\prime}$ cadrà poscia dove la $r^{\prime}$ s'incontra con la retta che, uscendo da $A$, si appoggia simultaneamente alle $p^{3}, r^{\prime}$.

Io. Sia $L$ un punto di $p^{3}$, e $\lambda^{5}$ la superficie del fascio $(\lambda)$ inerente al medesimo. La prima polare $\mu^{4}$ di $L$ rispetto a questa superficie è del quarto ordine e sega la superficie $\lambda^{5}$, a prescindere dalla $p^{3}$ contata due volte, lungo una curva del $\mathrm{I}_{4}{ }^{\circ}$ ordine con un punto $\mathrm{IO}^{\mathrm{plo}}$ in $L$.

Al variare di $L$ questa curva descrive una certa superficie $\sigma$ doppia per I. L'ordine della superficie $\sigma$ può aversi come segue: Una superficie

nei Rendiconti dell'Accademia deı Lincei, a. 1890-93 Una di talı superficie è rappresentabile univocamente sul piano mediante un sistema lineare $\infty^{3}$ di quartiche con I I punti fissi, quattro der quali sopra una retta ch'è imagine del punto triplo. Essa contiene, in generale, I I rette tutte corde della cubica, quattro delle quali passanti per il punto triplo, come si verifica infatti per ogni superficie $\lambda s$, dove le sette rette non contenenti il punto $L$ formano il luogo $0^{7}$. 
$\chi$ qualunque essendo involutora (autoconiugata) in $I$, le sue generatrici si corrisponderanno fra loro due a due $(\$ 9)$, e le coppie di generatrici coniugate formeranno un'muoluzione raziunale: visto che ale involuzione è segata sulla $\chi$ da un fascio generico di superficte $\chi$. Detta involuzione avrà quattro elementi doppi (uno essendo il genere della rigata $\chi$ ) il luogo dei qual, al variare $d_{i} \chi$, è appunto la superficie doppia $\sigma$. Ciascun punto di questa superficie sarà doppio per $I$. Infatti due generatrici coniugate $r$ ed $r^{\prime}$ sono segate dalle $\infty^{x}$ superficie autoconiugate $\lambda^{5}$ nelle $\infty^{1}$ coppie di punti coniugati; e $i$ due punti di ciascuna coppia coincidono allora ed allora soltanto, che coincidono le rette $r$ ed $r$.

Rappresentando, com'è sempre possibile, la congruenza $\delta$ delle corde d $1 p^{3}$ birazionalmente sui punti di un piano, in maniera che le $\infty^{2}$ schiere quadratiche di $\delta$ abbiano per imagini le $\infty^{2}$ rette del piano rappresentativo, vediamo che le $\infty^{2}$ rigate $\chi^{6}$ hanno per imagini le $\infty^{2}$ cubiche ellittche passanti per sette pitnti fissi $O_{1}, O_{2}, \ldots, O_{7}$, imagini delle sette corde $0^{7}$. Dunque l'involuzione, che la $I$ determina fra le $\infty^{2}$ corde di $p^{3}$, avrà per inagine una nuvoluzione piana del GeIser, descritta dalla rete di cubiche piane con sete punti fiss $O_{\mathrm{r}}, \ldots, O_{\overline{7}}^{*}$ ). Dunque la rigata doppia o aurà per imagine una sesiica $C_{O_{i}^{2}}^{6} O_{7}^{2}$ con sette punti doppi nei puntı fondamentali del prano rappresentativo, e sarà in conseguenza del $12^{\circ}$ ordine con la $p^{3}$ per direttrice sestupla e le $o_{1}, o_{2}, \ldots o_{7}$ per generatrici doppre.

II. L'intersezione del cono cubico osculatore ad una qualunque superficie $\lambda^{5}$ nel punto triplo $L$ con la cubica $p^{3}$ si compone di cinque punti cunulati in $L$ e di altri quatero $L_{1}, L_{2}, L_{3}, L_{4}$ : per la qual cosa le rette che uniscono quesii ultim punti con $L$ giacieranno sulla superficie $\lambda^{5}$. Dunque: ogni superficie $\lambda^{5}$ conttene quattro rette $L L_{2}$ $(i=1, \ldots, 4)$ usceriti dal suo punts triplo $L$ ed appoggate in un altro punto alla cubica $p^{3}$.

Ciascuna delle quattro rette è compresa nell'intersezione della superficie $\lambda^{5}$, cui essa appartiene, con la $\mu^{4}$, polare di $L$ rispetto alla medesima; ed è anche facile vedere che la retta $L L_{\imath}$ sarà conuıgata per

*) GeISer, Ueber qwei geometrische Probleme [CRelle's Journal, LXVII (i 867), pp. 78-89]. Vedi anche Bertini in Ann d1 Matem., vol. 8 (1877) e De Paolis, Le trasformazioni piane doppie nelle Mem. dell'Acc. dei Lincei, serie III, vol. $I^{\circ}$ ( 1877 ). 
intero ad un certo punto infintannente vicino al punto $L_{2}$ (nel quale essa si appoggia ulteriormente a $p^{3}$ ) e giacente sulla striscia infinitesima ch'è comune a tutte le superficie $\lambda$ lungo la cubica $p^{3}$; e precisamente su quella falda di $\lambda_{L}$ che non contiene la retta $L L_{i}$. [La quale striscia è anche comune a tutte le superficie $\varphi^{\prime}$ che, in forza $d_{i} I$, corrispondono ai piani $\varphi$ di $\Sigma$, superficie che, come vedremo fra poco ( $\$ I 2)$, devono toccare, con due delle tredici falde passanti per $p^{3}$, tutte le superficie $\lambda$ lungo la curva medesima].

Il sistema $\infty^{1}$ razionale delle prine polari $\mu^{4}$ è d'indice quattro. La superficie generata dai due sistemi proiettivi, uno (quello delle $\lambda^{5}$ ) d'ordine $s$ ed indice $I$, l'altro (quello delle $\mu^{4}$ ) d'ordine 4 ed indice 4 , è del $24^{\circ}$ ordine, e di essa fa parte la superficie $\sigma^{12}$. Il luogo delle rette analoghe ad $L L$, sarà quello che si ottiene escludendo dal luogo del $24^{\circ}$ ordine predetto la superficie doppia $\sigma^{12}$ : sarà dunque una superficie fondamentale, che chameremo $\alpha$, del dodicesino ordine. Del resto si potrebbe vedere anche direttamente che questa superficie fondamentale è del $12^{\circ}$ ordine, e contiene la $p^{3}$ come linea sestupla e le sette corde $o_{1}, o_{2}, \ldots, o_{7}$ come rette doppie. Delle sei falde con cui la superficie $\alpha$ passa per la cubica $p^{3}$, tre toccano l'una e tre l'altra delle due falde con cui le $\lambda^{5}$ passano per $p^{3}$.

I2. Se $L^{(\mathrm{r})}, L^{(2)}, L^{(3)}$ sono le tracce di un piano arbitrario sulla cubica $p^{3}$, 1 luogo dei punti coniugati esistentr in quel piano si spezzerà nelle tre quintiche da esso tagliate sulle superficie $\lambda^{(1)}, \lambda^{(2)}, \lambda^{(3)}$ e nella traccia del medesimo sulla superficie $\sigma^{\text {I2 }}$. Quindi: La trasformazione $I$ è del $27^{\circ}$ ordme, e le superficue $\varphi^{\prime}$, che essa fa corraspondere ai piani $\varphi$, passano con ireduce falde per $p^{3}$ e con cinque falde per ciascuna delle sette corde di $p^{3}$, che formano $o^{7}$.

Osservando che l'intersezione di una $\varphi^{\prime}$ con una superficie $\lambda$ consta soltanto di $p^{3}, o^{7}$ e della curva $l^{\prime}$ congunta ad una sezione piana $l \mathrm{~d} \lambda$ (curva ch'è del $16^{\circ}$ ordine), si ha che le $\infty^{3}$ superficie $\varphi^{\prime 27}$ devono toccare con due delie I 3 falde passante per $p^{3}$ le superficie $\lambda^{5}$ lungo tutta questa curva.

I3. Se le due falde comuni a tutte le $\lambda$ si toccano in un punto $L^{\prime}$ di $p^{3}$, il piano tangente comune intersechera la cubica ulteriormerte in un punto $L$ : sicchè la corda $L L^{\prime}$ giacierà per intero sulla superficie 
$\lambda_{L}$, è sarà coniugata a ciascuno dèi suoi punti; sarà insomma linea parassita in 1. Queste corde parassite, che chiameremo $b$, sono tante quanti i punti della $p^{3}$ nei quali si toccano le due falde comuni a tutte le superficie $\lambda$. Il numero di questi punti è otto, come subito si vede $(§ 4)$, quindi nella $I$ esistono otto corde $b_{\mathrm{r}}, \ldots, b_{8}$ parassite.

I4. Siano $L$ ed $M$ i punti dove i piani rispettivamente tangenti alle

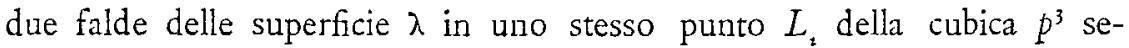
gano ulteriormente la cubica. Se una retta $\psi$ incontra la superficie $\alpha^{12}$ in un punto della generatrice $L_{3} M$, la curva $r^{\prime}$, ad essa coniugata, dovrà passare per quel certo punto $L_{\imath}$ della $p^{3}$, ch'è coniugato alla generatrice $L_{1} M$ di $\alpha$, e toccar quivi la retta $L_{1} L$. Ciascuno di questi incontri con direzioni assegnate implica dunque tre condizioni per il sistema delle curve $r^{\prime}$. Ne viene che la superficie $\alpha$ deve staccarsi tre volte dalla superficie Jacobiana delle $\varphi^{\prime}$; e che le superficie $\varphi^{\prime}$ devono oscularsi fra loro lungo tutta la cubica $p^{3}$ con ambedue le falde tangenti alle due falde delle superficie $\lambda$. Ciò è d'accordo col fatto che la superficie $\alpha$, staccandosi tre volte dalla detta Jacobiana, l'intorno della $p^{3}$, ch'è comune a tutte le $\varphi^{\prime}$, deve contare per tre curve fondamentali infinitamente vicine.

$\mathrm{La}$ base del sistema lineare formato da tutte le superficie $\varphi^{\prime 27}$ viene pertanto ad essere completamente nota. Infatti due $\varphi^{\prime}$ qualunque si segano, fuori delle linee fondamentali $p^{3}, 0^{7}$ ed $b_{1}$, in una curva $r^{\prime}$ del $27^{\circ}$ ordine, ch'è la corrispondente di una retta $r$ di $\Sigma$.

I5. Una superficie $\lambda^{5}$ è segata dal suo cono tangente in $L$ secondo una curva $l^{\text {I }}$ dell'undicesimo ordine, coniugata di $L$ : giacchè dall'intersezione totale di detta superficie si staccano le quattro corde $L L_{1}$, che hanno per luogo la superficie $\alpha^{\mathbf{1 2}}$ (\$ II). Ciò d'accordo col fatto che delle tredic falde con cui le $\varphi^{\prime}$ passano per $p^{3}$ undici sono variabilı e due fisse. La $l^{\text {II }}$ è raz:onale, e passa con otto rami per il punto $\dot{L}$. Variando $L$ su $p^{3}$, la $l^{x}$ descrive una superficue $\pi^{\prime}$ coniugata alla cubica fondamentale $p^{3}$ : essa è del $26^{\circ}$ ordne, contiene $p^{3}$ come curva multipla secondo otto ed $0^{7}$ come linea quintupla. Delle otto falde con cui la $\pi^{\prime}$ passa per $p^{3}$, quattro toccano l'uia e quattro l'altra delle due falde di una $\lambda^{s}$ qualsiasi.

16. Si consideri una qualsiasi $o_{2}$ delle sette corde $0^{7}$. La superficie $\omega_{1}^{\prime}$ coniugata ad $o_{2}$ sarà evidentemente il luogo della curva comune ad 
un piano passante per $o_{t}$ ed alla superficie $\lambda^{5}$ inerente al punto $L$, dove questo piano incontra ulteriormente la cubica $p^{3}$. Quindi ad ognuna delle sette corde di $p^{3}$ che compongono $0^{7}$ corrisponde una superficie $\omega_{2}^{\prime}$ del $6^{\circ}$ ordine contenente la $p^{3}$ e la o, come linee doppie e le altre sei corde di $p^{3}$, che assieme ad $o_{1}$ formano $o^{7}$, come linee semplici.

La Jacobiana del sistema delle $\varphi^{\prime 27}$ è ormai pienamente assegnata, componendosi della $\pi^{\prime 26}$, delle sette superficie $\omega_{i}^{\prime}(i=\mathrm{I}, 2, \ldots, 7) \mathrm{e}$ della superficie $\alpha$ contata tre volte.

Catania, novembre 1903.

Grazia Macrina Caldarera. 